\title{
Estudio de los Efectos del Ultrasonido en el Tejido Embrionario in vitro
}

\author{
Dr. José Grass $\mathrm{P}^{1}$. Sr. Luis Gaete $\mathrm{G}^{2}{ }^{2}$, Sr. Luis Hernández $\mathbf{M}^{2}{ }^{2}$, Sra. Marcela Fuenzalida B. ${ }^{3}$, \\ Sr. Julio Illánes $\mathbf{H}^{3}$. Sr. Dayid Lemus A.
}

\begin{abstract}
Chimeric chicken organs 'with 144 incubation hours, exposed to ultrasound during 10,20 and 30 minutes respectively, at $750 \mathrm{KHz}$ and $6.4 \mathrm{~W} / \mathrm{cm}^{2}$, were studied.

Histologycal findings are described, among them, the presence of necrosis in embrionic tissues irradiated for 20 munutes is remarked.

Histograms showed a significant decrease of mitosis in organs exposed by 10 and 20 minutes to ultrasound and an evident increase in those with 30 minutes of irradiation time.
\end{abstract}

El uso diagnóstico del ultrasonido se ha popula. rizado en la última década, haciéndose casi indis. pensable en algunas disciplinas tales como Obstetricia y Ginecología. También en Medicina Inter. na ha llegado a ocupar un importante lugar en el diagnóstico diferencial de las masas abdominales. Un uso terapéutico que aprovecha las cualidades físicas del ultrasonido como es el aumento local de temperatura, se emplea en Ortopedia desde hace algunas décadas.

Las ondas ultrasónicas, al igual que otras ondas acústicas se producen cuando un material es puesto en vibración, es decir, sus partículas oscilan periodicamente en torno a su posición de equilibrio. Cuando la frecuencia de vibración de una onda es superior a 20.000 oscilaciones por segundo, que es la mayor frecuencia perceptible por el aído humano normal, se llama onda ultrasónica ${ }^{13}$.

Existen diversos tipos de generadores de ultrasonido, los más usados son los cristales dé cuarzo y cerámicas construidas con una aleación de Titana. to-Zirconato de plomo y bario con adición de tierras raras. Estos materiales tienen la propiedad de cambiar sus dimensiones físicas cuando se les

1 Médico Instituto Traumatológico, Depto. Cirugía Facultad de Medicina, Universidad de Chile.

2 Investigadores Depto. de Física, Facultad de Ciencias, 3niversidad de Santingo de Chile.

3 Investigadores Laboratorio de Embriología Experimental, Depto. Morfología Experimental, Facultad de Medicina, Universidad de Chjle.

Investigación financiada parcialmente con el proyecto B No 1401-8325 del Depto. de Desarrollo de Investigaciones de la U. de Chile; y el subprograma 08-09-82 23 del DYCIT, U. de Santingo de Chile. aplica una tensión eléctrica (o viceversa). Si el voltaje aplicado es alterno, las piezas de cerámica o cuarzo aumentan y disminuyen alternativamente de espesor, originando una onda elástica de frecuencia igual a la del voltaje aplicado.

Como otras ondas energéticas empleadas por el hombre, el ultrasonjdo es potencialmente daffino, dependiendo de la intensidad y duración de la exposición a la que es sometido el tejido ${ }^{5} \cdot 11$. 19.

El análisis de la literatura muestra numerosos trabajos que comunican, por ejemplo, rotura cromosómica ${ }^{B}-18 \cdot 27$, retardo en el trecimiento celular ${ }^{17}-22$, daño en tejido nervioso de diversos animales $5 \cdot 7 \cdot 26 \mathrm{y}$ alteraciones en otros parénquimas diversos $1 \cdot 12 \cdot 20$.

En general muchos de los trabajos son contradictorios ${ }^{11} \cdot{ }^{17}$, no habiéndose demostrado aún alguna relación significativa entre irradiación intra. uterina y anormalidades en la especie humana mediante evaluaciones clínicas hasta el año de edad $^{24}$.

Interesados en investigar los posibles efectos del uitrasonido sobre células en activo proceso de diferenciación y crecimiento, período en el cual los elementos celulares se hacen fuertemente susceptibles a las variaciones ambientales, hemos elegido al esbozo del miembro de pollo. El desarrollo de este órgano en el campo de la Biología Experimental representa un excelente modelo, que permite abordar variados problemas, entre ellos los relacionados con la clínica $9 \cdot 21-25 \cdot 29$.

Durante la diferenciación tanto en situ como in vitro del esqueleto apendicular, se establecen complejas interacciones de tejidos, cuyos mecanismos íntimos son desconocidos $-9-21-25-29$, pero 
que se manifiestan morfológicamente en actividad mitótica del mesoderma y migración de células, que originan elongación del esbozo, para ser seguido de condensación del mesoderma que se diferencia a los distintos tejidos componentes del miembro.

\section{MATERIAL Y METODOS}

Embriones de pollo (Gallus gallus) fueron empleados en el trabajo, determinando su estado de desarrollo según la tabla de Hamburger y Hamilton 10 . Después de 96 hrs. de incubación, los huevos fueron intervenidos, procedjéndose a extraer los embriones, que fueron acumulados en un pocillo que contenía líquido de Tyrode con antibióticos y antimicóticos.

Una vez despejados los embriones de sus envol. turas, se procedió a microdisecar los esbozos de los 4 miembros y transferirlos con micropipetas a una solución de suero equino más líquido Tyrode en proporción $1: 2$ y mantenidos a $4^{\circ} \mathrm{C}$.

Los receptores para los esbozos de miembro fueron embriones de pollo con 8 dias de incubación. Este material fue preparado según el método de Zwilling ${ }^{29}$, extrayendo albúmina de huevo $y$ practicando una ventana en la cámara. A continuación se aspiraron con micropipetas los esbozos de miembros y se depositaton sobre un filtro millepore de $5 \mathrm{~mm}$, de diámetro; $0,45 \mu \mathrm{m}$ de poro y 125 $\mu \mathrm{m}$ de grosor.

Finalmente, con micropinzas los filtros conteniendo los explantes fueron invertidos y aplicados directamente sobre los capilares alantocoriónicos del receptor. Realizada esta maniobra, se procedió a sellar la ventana con tela adhesiva transparente $y$ se levaron los huevos a la cámara de cultivo donde fueron mantenidos durante $48 \mathrm{hrs}$. A continuación los esbozos fueron sometidos a irradiación ultrasó. nica durante 10,20 y 30 minutos respectivamente $y$, mantenidos posteriormente otros 5 días en incubación, para ser extraidos y fijados en DubosqBrasil, incluídos, cortados y tenidos con hematoxilinaeosina y Acido periódico de Schiff (PAS).

Los tejidos fueron analizados con microscopía ocular y se registró el número de mitosis en un área de $25.880 \mu \mathrm{m}^{2}$. Con los datos obtenidos, se confeccionó un histograma que fue evaluado me. diante la prueba de Student.

Para irradiar los tejidos estudiados se utilizó un dispositivo construido expresamente para este experimento. Consiste básicamente en un sistema electrónico de excitación compuesto de un oscilador, un amplificador de potencia y un transductor cuyo elemento activo es una cerámica de titanato de bario (PZT4) alojada en un soporte de latón que le brinda su protección mecánica y eléctrica. El sistema trabaja en sintonía a una frecuencia de $750 \mathrm{KHz}$.

El dispositivo experimental empleado en la presente investigación, descrito brevemente en el párrafo anterior y mostrado en la Fig. 1, no permite medir directamente la intensidad acústica presente en los tejidos sometidos al campo ultrasónico. Sin embargo, es posible hacer un cálculo de ésta a partir de los parámetros de emisión determinados durante la realización del experimento. En efecto, la potencia radiada por el transductor depende de la potencia eléctrica consumida por el elemento radiante y de su capacidad de conversión de ener. gía eléctrica a mecánica (rendimiento).

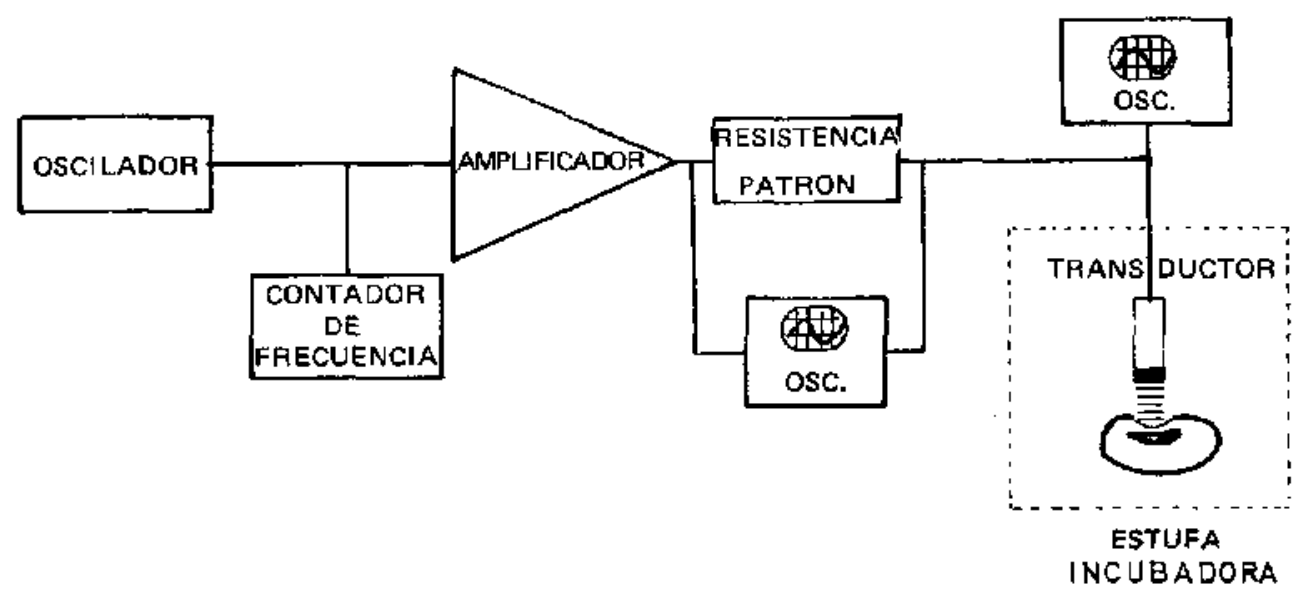

Figura $\mathbf{L}$.

Esquema del sistema experimental 
La primera se obtiene de la expresión 13. $^{3}$.

$$
\mathrm{W}=\mathrm{i} \mathrm{v} \cos \phi
$$

donde $W$ es la potencia eléctrica consumida por el elemento radiante, $i$ es la corriente que consume el transductor, $v$ es la tensión aplicada $y \phi$ es el ángulo de desfase entre " $i$ " $y$ " $v$ ".

Para nuestra situación experimental tenemos que

$$
\begin{aligned}
& i=4.56 \text { mAmp } \\
& v=7,6 \text { wolts } \\
& \phi=44^{\circ}
\end{aligned}
$$

lo que da una potencia consumida de 24 mWatts, pero considerando que la eficiencia de una cerámica montada en esta forma es del orden del $40 \% 12$ $y$ que ésta radia tanto hacia adelante como hacia atrás, la potencia efectiva sobre los tejidos es de aproximadamente $5 \mathrm{~mW}$ att .

La frecuencia empleada fue de $750 \mathrm{kHz}$ y la superficie radiante de la cerámica de $0,78 \mathrm{~cm}^{2}$, siendo por lo tanto de este grosor el haz de ultrasonido que se proyectó sobre el esbozo del miembro.

La intensidad acústica, que corresponde a la cantidad de energía por segundo que fluye a través de cada unidad de superficie, fue entonces de 6.4 $\mathrm{mWatt} / \mathrm{cm}^{2}$.

\section{RESULTADOS}

El anailisis macroscópico de los esbozas controles no mostró diferencia de crecimiento con respecto a los irradiados, tampoco se observaron diferencias entre aquellos sometidos a distintos tiempos de exposición.

En general todos los esbozos presentaron al momento de su fijación diferencia de esbozos car. tilaginosos, musculatura e incluso formación de algunos fanéreos como plumas. En el tejido some. tido a 10 minutos de irradiación sólo llama la atención la presencia de algunos cuerpos multilaminillares (Fig. 2). Aquellos irradiados por $20 \mathrm{mi}$ nutos muestran al examen microscópico algunos núcleos condensados de tejido mesenquimático, rico en mucopolisacáridos acidos e intensa infiltración de leucocitos polimorfonucleares y eosinófilos. Destaca la presencia de zonas de intensa necrósis (Fig. 3), en donde no es posible identificar estructuras celulares definidas, $y$ en algunos casos se observa el mesénquirna del alantocorion con gran proliferación de elementos fibrilares y celulares, entre ellos células mesenquimáticas, eosinófilos y otros elementos figurados. El limite entre el mesénquima alantocoriónico y la zona de prolife. ración fibrilar es neta y clara, distinguiéndose tam. bién gran riqueza de capilares.

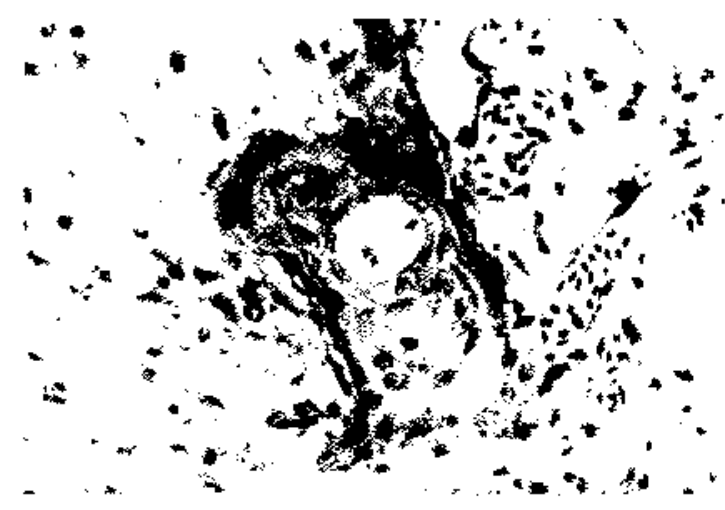

Figura 2.

Esbozo de mjembro sometido a irradiación ultrasónica de $6.4 \mathrm{mw} / \mathrm{cm}^{2}$ durante 10 minutos, en el que se aprecia la presencia de cuerpos multilaminillares

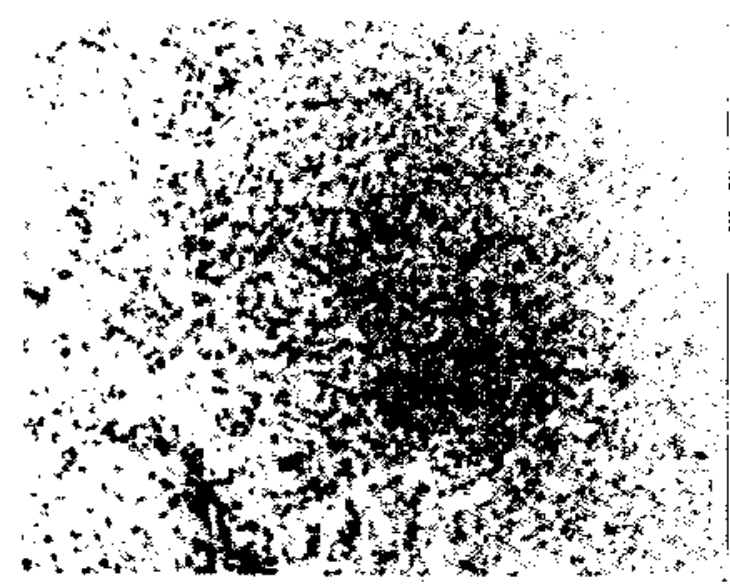

Figura 3.

Esbozo de miembro de pollo irradiado por 20 minutos, con presencia de un foco de necrosis que impide la clara identificación de los elcmentos celulares.

El análisis estadístico del número de mitosis observada en $26.880 \mathrm{\mu m}^{2}$ de tejido, muestra resul. tados interesantes. En efecto, los tejidos control mostraron un promedio de $4.11 \pm 0.46$ mitosis en 44 registros, lo que supera significativamente los irradiados durante 10 y 20 minutos, que presenta. ron $2.35 \pm 0.30$ mitosis, en 48 registros y $3.24 \pm$ 0.28 en 45 registros respectivamente. Aquellos tejidos irradiados durante 30 minutos, presentaron un significativamente alto número de mitosis en el área indicada, $6.91 \pm 0.39$ para 84 observaciones (Fig. 4).

La tinción de PAS demostró gran cantidad de mucopolisacáridos neutros y glicógeno en las células epiteliales y membranas basales. 


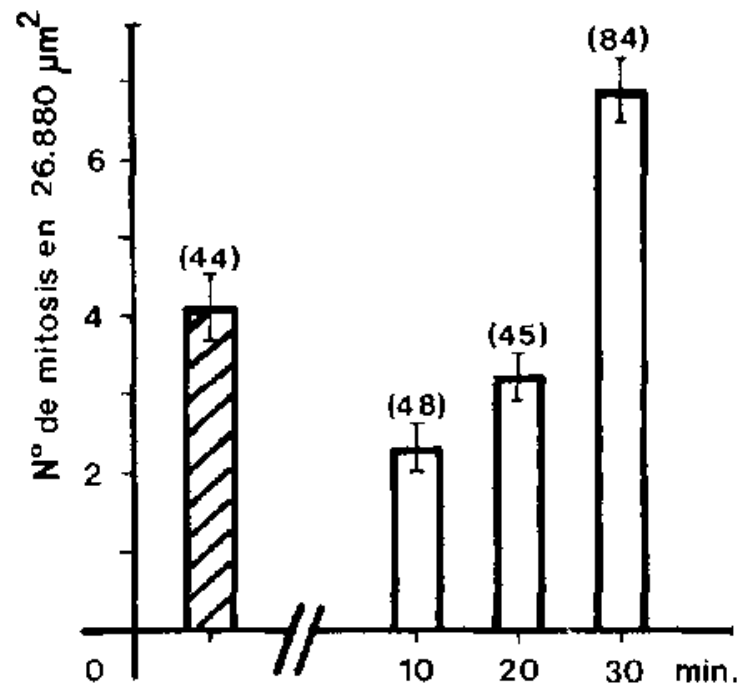

Figura 4.

Estudio comparado del indice mitótico en esbozos de miembros sometidos a diferentes tiempos de irradiación ultrasónica.

La barra achurada representa los controles y el número entre paréntesis representa cl número de observaciones.

El Test de Student calculado para los controles versus los irradiados y entré éstos fue de $\mathrm{P}<0.05$.

\section{DISCUSION}

Entre los hechos que más laman la atención en el estudio efectuado. está la presencia de focos necróticos, en la periferia de los cuales puede observarse proliferación fibrilar de límites netos que continúa externamente con un tejido conjuntivo típico (Fig. 3). Esta necrosis puede ser atribuida a isquemia del esbozo, por incapacidad del alantocorion para aportarle la irrigación adecuada; pero esta hipótesis puede ser descartada por la evidencia de gran proliferación de capilares en torno al foco de necrosis. Otra posibilidad, que a nuestro juicio es más acertada, consiste en atribuir la necrosis a la irradiación ultrasónica; apoyan esta teoría estudios efectuados en oido de gato por Barnett ${ }^{1}$, que muestra daño histọlógico y neurofissiológico en el laberinto vestibular, con necrosis de algunas células y desorganización de otras al aplicar durante 20 minutos intensidades de $5-10 \mathrm{~W} / \mathrm{cm}^{2}$ con 3.5 MHz. Otros trabajos efectuados en cerebros de gato4.14, muestran necrosis focal periférica rodeando islotes de tejido sano con irradiación de 3082 a $9655 \mathrm{~W} / \mathrm{cm}^{2}$ con una frecuencia de $1 \mathrm{MHz}$; y en ratas se ha encontrado necrosis en los ganglios espinales y hueso, con daño en la médula espinal ${ }^{26}$ aplicando $1 \mathrm{~W} / \mathrm{cm}^{2}$ con $2 \mathrm{MHz}$ desde 80 a 200 segundos. Debe considerarse dentro del análisis, que estos tejidos descritos son de origen diferente al estudiado en nuestro trabajo, como así también, los tiempos de exposición y dosis empleadas.

El mecanismo por el cual el ultrasonido sería capaz de lesionar los tejidos no está claro; se ha postulado un efecto térmico, dado el aumento de temperatura descrito como variable entre $5,5 \mathrm{y}$ $11^{\circ} \mathrm{C}^{7} \cdot 26$ dependiendo de las intensidades y tiempos empleados. Para esta acción parece ser necesario la lidratación normal de la célula, cumo lo demuestra uni estudio efectuado en bacterias secas liofilizadas ${ }^{2 \sigma}$, que son incapaces de absorber suficiente energía para ser destruidas. Otros autores postulan que la agitación mecánica inducida por el ultrasonido en la célula origina los daños observados y no el aumento de temperatura, pues al someter el tejido a un alza térmica igual a la ocurrida durante la irradiación, no se observan alteraciones estructurales ${ }^{2}$.

No puede descartarse que las alteraciones morfológicas encontradas sean ocasionadas por daño en el material genético de algunas células en multiplicación ${ }^{3}-11 \cdot 17 \cdot 18 \cdot 19$, que pudiera alterar alguna proteina o enzima vital en su descendencia, haciéndolas no viables a corto o mediano plazo. En células $\mathrm{He} \mathrm{La}$ irradiadas no se han encontrado evidencias de alteraciones de la síntesis protéica, pero no ha podido excluírse inhibición de la sintesis de fracciones protéicas menores ${ }^{17}$, por lo que hubiera sido de gran utilidad efectuar un estudio electroforético de las proteinas componentes de] esbozo de niembro.

La alteración en la información genética también puede postularse conno causa de la reacción proliferativa por parte del mesénquima y en las diferencias encontradas en la cantidad de cartilago, en los distintos tiempos de exposición.

La ausencia de alteraciones morfológicas significativas en el tejido irradiado durante 10 minutos puede atribuirse al escaso tiempo de exposicion, lo que influye en los resultados junto a la frecuencia e intensidad de la onda $5 \cdot 11^{\cdot} \cdot 19$. Por otra parte, las alteraciones morfológicas como expresión fenotípica de una alteración genotipica inducida es un evento raro, encontrándose por lo general en menos de una célula por mil expuestas a alguna noxa ${ }^{3}$ - 23, lo que también es aplicable a la radiación ultrasónica, encontrándose a veces muchas células normales a la observación con microscopio óptico, con graves alteraciones en su superficie topográfica al examen con microscopio electrónico ${ }^{16}$.

Difícil es la interpretación de los resultados estadísticos de la contabilización de las mitosis. En efecto, a pesar que los esbozos irradiados presentaron un aumento de su tasa de mitosis en directa relación con el tiempo de exposición (Fig. 4), aquellos irradiados por 10 y 20 minutos muestran 
un registro de índices mitóticos significativamente menores a los controles, ocurriendo precisamente lo contrario en los 30 minutos. Podría plantearse, a la luz de estos resultados preliminares, que el efecto de la irradjación ultrasónica sobre esbozos de miembros cultivados in vitro, produciría un efecto de aceleración en el índice mitótico, a partir de un tiempo de exposición crítico, que en nuestro caso sería de 30 minutos y que en tiempos inferiores a éste, produciría un bloqueo en la velocidad normal de división celular. Estas conclusiones sólo son válidas para el tejido estudiado en este trabajo y ante iguales parámetros de emisión, pues se ha encontrado disminución de la actividad mitótica en cultivo de células $\mathrm{B}$ alb/e 3T3, clon 1-13 sometidas durante 30 minutos a $15 \mathrm{~mW} / \mathrm{cm}^{2} 26$ entre otros tejidos diversos ${ }^{14}-22$, mientras que en cultivos de leucocitos humanos irradiados con 22 $\mathrm{mW} / \mathrm{cn}^{2}$, durante una hora aumenta la multiplicación celular ${ }^{19}$

En relación a la intensidad acústica, podemos notar que la empleada por nosotros $\left(6.4 \mathrm{~mW} / \mathrm{cm}^{2}\right)$ es inferior al unbral dado por Macintosh ${ }^{19}$, quien sólo encontró daño cromosómico sobre 8.2 $\mathrm{mW} / \mathrm{cm}^{2}$; pero la variedad de datos contradictorios que aporta la bibliografía no permite concluir nada definitivo $3 \cdot 26$

Aún no es posible extrapolar nuestros resultados obtenidos en el laboratorio a la situación clinica: pues el valor de los hallazgos sólo es válido para la frecuencia, tiempo de exposición y técnicas empleadas en este tipo de tejidos. Sin embargo, parece prudente que en el uso diagnóstico y terapéutico del ultrasonido deben estimarse los beneficios y los riesgos posibles, teniendo cuidado en la elección del sitio de aplicación tanto como en la duración de ésta.

\section{RESUMEN}

Esbozos de miembros de ave con 144 horas de incubación fueron sometidos a irradiación ultrasónica con una frecuencia de $50 \mathrm{kHz}$ y una intensidad de $6.4 \mathrm{~mW} / \mathrm{cm}^{2}$ durante 10,20 y 30 minutos respectivamente.

Se describen los hallazgos histológicos, entre lo que llama la atención la presencia de focos de necrosis en aquellos irradiados durante 20 minutos. El histograma mostró disminución significativa de las mitosis en los de 10 y 20 minutos, y aumento notorio en el de 30 minutos.

Se compara lo encontrado con lo descrito por la literatura.

\section{REFERENCIAS}

I Barnett S. B. The effect of uitrasonic irradiation on the structural integtity of the inner ear labyrith. Acta Otolaryngol 89: 424, 1980.

${ }^{2}$ Barnett S. B. The influence of ultrasound and temperature on the cochlear microphonic response following a round window irradiation. Acta Otolaryngol. 20:32, 1980 .

${ }^{3}$ Barret $J, C, T s o$ PO, Evidence for the progressive nature of neoplasic transformation. PNAS. USA 75: 37621,1978

4 Bernard J. W. et al. Small localized ultrasonic lesions in the white and gray matter of the cat brain. Arch Neural Psychiatry 75: 15, 1956.

${ }^{5}$ Dunn F., Lohnes J. E. Fry F. J. Frequency dependence of thresiold ultrasonic dosages for irreversible structural changes in mammalian brain. J. Acous. Soc. Am. 58: 512, 1975.

- Fleischmajer R., Billigham $R$. EphithelialMesenchymal Interactions 18 th Hahnemann Symposium, Ed. Williams and Wilkins Ca., Baltimore 1968 .

7 Fry $F, f$. et al. Transkull focal lesions in cat brain produced by ultrasound. J. Neurosurg 54: 659, 1981.

${ }^{8}$ Galperin-Lamaitre $H_{\text {, }}$ et al. Fragmentation of purjfied mamalian DNA molecules by ultrasound below human therapeutic doses, Humangenetik 1: 61, 1975.

${ }^{9}$ Gumpet-Pinat M. Ectoderm and mesoderm interactions in the limb bud of the chick ernbryo studied by transflter cultures: cartilage differentiation and ultrastructural observation. J. Embrio Exp Morphol. 59: 157,1980 .

10 Homburger V. and Hamilton H. L. A series of normal stages in the development of the chick embryo. J. Morphol, 88: 49, 1951

11 Harkányi Z. et al. A search for and effect of ultrasound afone and in combination with $X$ rays on chromosomes in vivo. British J. Radiol $51: 46,1978$.

12 Hueter T. F., Bolt R. H. Sontcs, Ed. John Wiley and Sons. Med., New York, 1965.

13 Kinsler L. E., Frey A. R. et al. Fundamentals of acoustics $3^{\circ} \mathrm{Ed}$., John Wiley and Sons, New York, 1982.

14 Kremkau F. W. Witcofski R. L. Mitotic reduction in rat liver exposed to ultrasound. J. Clin Ultrasound 2: 123,1974 .

is Lemus D., Bahamonde L., De la Barrero C., Musz C. Comportamiento in vitro de esbozos de mienbros en presencia de un campo electromagnético. $X$ Cong. Chil. Ortop. Traumatol, Vina del Mar 24 26, nov. 1982.

16 Liebesking D. et al. Morphological changes in the surface chatacteristics of cultured cells after exposure to diagnostic ultrasound. Radiology 138: 419, 1981.

17 Liebesking $D$. ef al, Diagnostic Ultrasound: Effects on the DNA and Growth Patterns of animals cells. Radiology 131: 177, 1979 .

18 Macintosh $I . J . C$. Chromosome aberrations induced by an ultrasonic fetal pulse detector. Br. Med. J. 4: $92,1970$.

19 Aocintosh I. J. C., Davey D. A. Relationship between intensity of ultrasound and induction of chromosome aberrations. Br. J. Rađiol, 45 : 320, 1972.

${ }^{20}$ O'Brien W. D. et al. Morphological changes to mouse testicular tissue from in viץo ultrasonic irradiation. Ultrasound Med. Biol. 5: 35, 1979.

21 Prutov M. P. and Kiney M. Interaction ecto-mesodermique dans l'establissement de la polarite dorso-ventrale du pied de l'embryon de poulet, C. R. Aca. Sci. Ser. O. $277: 1225,1973$. 
22 Pizzarello D. J. et al. Effect of pulsed low power ultrasound on growing tissues, I. Developing mamma. lian and insect tissues. Exp. Cell. Biol. 46: 179, 1978.

23 Sexholm $H . J$. $K$. The oncogenic potential of three different 7.12 - dimenthylbenz-anthracene transformed $\mathrm{C} 3 \mathrm{H} / 10 \mathrm{~T}-1 / 2$ cell clones at various passages and the importance of the mode of inmunosuppresion. Eur. J. Cancet 15: 515, 1979.

24 Sheidt $P$, et al. One year follow up of infant exposed to ultrasound in utero. Am. J. Obstet. Gynecol, $131: 743,1978$.

25 Searls R. I., Smith A. A. Evidence that ectoderm influences the differentiation of muscle in the limbs of the embrionic chick. J. Exp. Zool., 220: 343, 1982.

26 Stokenberg $S$. $J$. et al. Toxic effects of ultrasound in mice: Damage to central and autonomic nervous system. Toxicology and Applied Faramacology 53: $432,1980$.

27 Taylor $K . J$. W. Current status of toxicity investigations. J. Clin. Ultrasound 2: 149, 1974.

28 Vela $G . R$. Wh J. F. Mechanizm of lethal action of $2.450 \mathrm{MHz}$ radiation on microorganisms. Applied and Environmental Microbiology 37(3): 550, 1979.

${ }^{29} Z$ willing $E$. A modified choriollantoic grafting procedure. Transplant Bull., 6: 238, 1959. 